\title{
Empirical Wavelet Transform Based Approach for Extraction of Fundamental Component and Estimation of Time-Varying Power Quality Indices in Power Quality Disturbances
}

\author{
G. Ravi Shankar Reddy ${ }^{1}$ and Dr.Rameshwar Rao ${ }^{2}$ \\ ${ }^{1}$ Dept.of ECE, CVRCollege of Engineering, Hyderabad, India \\ ${ }^{2}$ Ex-Vice Chancellor, JNT University, Hyderabad, India \\ ${ }^{1}$ ravigosula_ece39@yahoo.co.in, ${ }^{2}$ rameshwar_rao@hotmail.com
}

\begin{abstract}
In this paper Application of an Empirical Wavelet Transform based technique is proposed to estimate time-varying $P Q$ indices for accurate assessment of Power Quality Disturbances. The EWT approach mainly aims to extract the actual fundamental frequency component and disturbance components from any distorted signal. The empirical wavelet transform consists of two major steps: detect the Fourier supports, and build the corresponding wavelet accordingly to those supports; filter the input signal with the obtained filter bank to get the fundamental component and disturbance components. Since the extracted components contain only one frequency component, Hilbert transform is utilized to estimate the instantaneous frequency and amplitude information, from this information we can estimate time-varying $P Q$ indices. The proposed method is employed to assess successfully all sorts of Power Quality Disturbances such as voltage sag, swell, interruption, transients, harmonics, spikes, notches etc. From the results we can say that the proposed method detects disturbance start time, end time, duration of existence and its content more accurately.
\end{abstract}

Keywords: Empirical Wavelet Transform, time-varying Power Quality Indices, Power Quality Disturbances, fundamental frequency Component, Fourier supports, Filter bank

\section{Introduction}

Power quality signal detection and its classification is of great importance in industrial and commercial power system. The disturbances in power quality signal are threats for electronic equipments which are sensitive to these disturbances [1].Commonly these types of disturbances like swell, sag, and harmonics are referred to as the pollutions in power grid. The main sources include SMPS in computers, rectification circuits, non-linear resistive loads, inverters and verities of switching circuits, rotating machineries etc.

The real-time measurement of frequency is now important for many applications in the power system. Several methods have been proposed and adopted to compute frequency for the power system applications. The Fast Fourier Transform is the most widely used method for frequency estimation. Due to leakage effect, and aliasing effect, it cannot produce a better result [2-3]. Further extensions and enhancements are done on this method by utilizing the original FFT with different windowing and interpolation to produce an accurate estimate [4]. Various techniques for detection and classification of power quality disturbances have been proposed recently [5-6]. Mainly it includes hidden Markov models, artificial neural networks, Wavelet transform for the analysis of harmonic pollution etc. Wavelet transform based processing of power quality disturbances is explained in [7].

The wavelet analysis is one of the most useful tools in numerous applications. Although the wavelet packets, Malvar-Wilson wavelets, Brushlets and synchro squeezed 
wavelets attempted to build adaptive wavelet based filter banks, they performed as partially adaptive wavelet transforms due to prescribed schemes [7].

S-Transform is another extensively used time-frequency analysis tool because of its frequency-dependent window and hence used for estimation of PQ indices. The ST has many variants based on the window selected, its shape and domain of analysis .One such approach is recently proposed Fast Discrete-Transform (FST) [8].The statistical signal processing techniques like prony ,Multiple Signal Classification(MUSIC),Estimation of Signal Parameters via Rotational Invariance Technique(ESPIRIT) and few hybrid techniques [9] based on these are known for the accurate estimation of signal parameters even in the presence of noise. The other techniques, explored for PQ assessment are an artificial neural network, Kalman filtering, time-frequency-distribution technique. Considering the advantage of FFT, efforts have been made for the development of new methods based on FFT for enhanced performance and robustness to analyze the nonstationary signals.

Recently, a new approach, Empirical Wavelet Transform (EWT) [10], has been proposed to build a family of adaptive wavelets capable of extracting different components of a signal. This method has an advantage of adaptability according to the analyzed signal and can isolate the different modes of the signal. Tracking of instantaneous power quality (PQ) indices is very essential for better characterization of time-varying voltage and current signals. An attempt has been made in this paper to utilize the self-adaptiveness of the EWT in estimating the fundamental frequency component in Power Quality disturbances and there by the estimation time-varying PQ indices.

The organization of this paper is as follows, Section 2 presents a brief review of EWT technique required to analyze the signal. Hilbert Transform is discussed in Section 3. In Section 4, Time-Varying power Quality indices are presented. Section 5 considers several test signals and the time-varying PQ Indices are calculated using EWT. This section also provides results which exhibit the effectiveness of this technique. Finally, conclusions are drawn in Section 6.

\section{Empirical Wavelet Transform}

EWT is a recently proposed method to adaptively detect the different modes of the signal and consequently construct the empirical wavelets to represent the signal by different modes detected. Empirical wavelets means constructing a set of wavelets adapted to the processed signal, i.e., in Fourier domain means constructing a set of bandpass filters [10-11]. Adaptation here lies in detecting filter supports according to the information located in the processed signal. Modes can be viewed as the principal components (referred to as amplitude modulated and frequency modulated (AM-FM) components) of the signal which represents the signal completely

The adaptableness in this transform is provided by the segmentation of Fourier axis is done in away so as to separate different portions of the spectrum which correspond to modes that are centered around a specific frequency and of compact support. To find such boundaries we find the local maxima's in the Fourier spectrum [10].

Consider a real signal $x(n)$, which is sampled at a frequency of $f_{s}$. First apply the FFT to the discrete signal $x(n)$ to find the frequency spectrum $X(\omega)$ and then obtain the set of maxima $\delta=\left\{\delta_{n}\right\}_{n=1,2, \ldots ., N}$ in the Fourier spectrum by means of the magnitude and frequency distance thresholds and infer their corresponding frequency $\Omega_{n}$. Here $\mathrm{N}$ is the number of frequency components assessed using FFT. Now with this set of frequencies $\Omega=\left\{\Omega_{n}\right\}_{n=1,2, \ldots, N}$ corresponding to maxima, the Fourier spectrum $\left[0, f_{S} / 2\right]$ is segmented into $\mathrm{N}$ segments, where each segment is defined as $\Lambda_{n}=\left[\omega_{n-1}, \omega_{n}\right]$.Assuming $\omega_{0}=0$ and 
$\omega_{N}=f_{S} / 2$, the boundaries $\omega_{n}$ are obtained as given in(1),representing the center of two successive maxima

$$
\omega_{n}=\frac{\Omega_{n}+\Omega_{n+1}}{2} \quad \text { for }, 1 \leq n \leq N-1 .
$$

Where $\Omega_{n}$ and $\Omega_{n+1}$ are the frequencies, and $\omega_{n}$ is their corresponding boundary. Then, the Fourier segments will be $\left[0, \omega_{1}\right],\left[\omega_{1}, \omega_{2}\right], \ldots \ldots .,\left[\omega_{N-1}, f_{S} / 2\right]$.After attaining the set of bounds $\omega=\left\{\omega_{n}\right\}_{n=1,2, \ldots . . N-1}$, we designate $\omega_{n}$ to be the limits between each segment $\left(\omega_{0}=0\right.$ and $\left.\omega_{N}=\pi\right)$.Each segment is denoted $\Lambda_{n}=\left[\omega_{n-1}, \omega_{n}\right]$, then it is easy to see that $\bigcup_{n=1}^{N} \Lambda_{n}=[0, \pi]$.centered about each $\omega_{n}$, we define a transition phase $\mathrm{T}_{n}$ of width $2 \tau_{n}$.A bank of $\mathrm{N}$ wavelet filters, consist of one low-pass filter and N-1 band-pass filters are defined based on the well-detected boundaries [12-13].

The empirical wavelets are defined as bandpass filters on each $\Lambda_{n}$ and based on $\Lambda_{n}$ a wavelet tight frame be able to defined, to do so, we use the notion used in the building of both Littlewood-Paley and Meyer's wavelets [10], a wavelet tight frame $B=\left\{\left\{\phi_{n}(t)\right\}_{n=1}^{N-1},\left\{\psi_{n}(t)\right\}_{n=1}^{N-1}\right\}$ is defined. And $\forall n>0$, their Fourier transforms, i.e., the empirical scaling function $\hat{\phi}_{n}(\omega)$ and the empirical wavelets $\hat{\psi}_{n}(\omega)$ by expressions of (2) and (3), respectively.

$$
\hat{\phi}_{n}(\omega)=\left\{\begin{array}{l}
1 ; \text { if }|\omega| \leq \omega_{n}-\tau_{n} \\
\cos \left[\frac{\pi}{2} \beta\left(\frac{1}{2 \tau_{n}}\left(|\omega|-\omega_{n}+\tau_{n}\right)\right)\right] \text { if } \omega_{n}-\tau_{n} \leq|\omega| \leq \omega_{n}+\tau_{n} \\
0 ; \text { otherwise }
\end{array}\right.
$$

and

$$
\hat{\psi_{n}}(\omega)=\left\{\begin{array}{l}
\text { if } \omega_{n}+\tau_{n} \leq|\omega| \leq \omega_{n+1}-\tau_{n+1} \\
\cos \left[\frac{\pi}{2} \beta\left(\frac{1}{2 \tau_{n+1}}\left(|\omega|-\omega_{n+1}+\tau_{n+1}\right)\right)\right] \text { if } \omega_{n+1}-\tau_{n+1} \leq|\omega| \leq \omega_{n+1}+\tau_{n+1} \\
\sin \left[\frac{\pi}{2} \beta\left(\frac{1}{2 \tau_{n+1}}\left(|\omega|-\omega_{n+1}+\tau_{n+1}\right)\right)\right] 0 ; \text { if } \omega_{n}-\tau_{n} \leq|\omega| \leq \omega_{n}+\tau_{n} \\
0 ; \text { otherwise }
\end{array}\right.
$$

The function $\beta(x)$ is an arbitrary function such that

$$
\beta(x)=\left\{\begin{array}{l}
0 ; \text { for }, x \leq 0 \\
\beta(x)+\beta(1-x)=1 \forall x \in[0,1] \\
1 ; \text { for }, x \geq 1
\end{array}\right.
$$

Many functions satisfy these properties, the most used in the literature [9] is 


$$
\beta(x)=x^{4}\left(35-84 x+70 x^{2}-20 x^{3}\right)
$$

Regarding the choice of $\tau_{n}$, several choices are possible. The simplest is to select $\tau_{n}$ proportional to $\omega_{n}: \tau_{n}=\gamma \omega_{n}$ where $0<\gamma<1$. To meet the requirement of tight frame [9], the parameter $\gamma$ must fulfill the following equation:

$$
\gamma<\min _{n}\left(\frac{\omega_{n+1}-\omega_{n}}{\omega_{n+1}+\omega_{n}}\right)
$$

Therefore, $\forall n>0,(2)$ and (3) simplify to (7) and (8)

$$
\hat{\phi}_{n}(\omega)=\left\{\begin{array}{l}
\text {; if }|\omega| \leq(1-\gamma) \omega_{n} \\
\cos \left[\frac{\pi}{2} \beta\left(\frac{1}{2 \gamma \omega_{n}}\left(|\omega|-(1-\gamma) \omega_{n}\right)\right)\right] ; \text { if }(1-\gamma) \omega_{n} \leq|\omega| \leq(1+\gamma) \omega_{n} \\
0 ; \text { otherwise }
\end{array}\right.
$$

and

$$
\hat{\psi_{n}}(\omega)=\left\{\begin{array}{l}
1 ; \text { if }(1+\gamma) \omega_{n} \leq|\omega| \leq(1-\gamma) \omega_{n+1} \\
\cos \left[\frac{\pi}{2} \beta\left(\frac{1}{2 \gamma \omega_{n+1}}\left(|\omega|-(1-\gamma) \omega_{n+1}\right)\right)\right] ; \text { if }(1-\gamma) \omega_{n+1} \leq|\omega| \leq(1+\gamma) \omega_{n+1} \\
\sin \left[\frac{\pi}{2} \beta\left(\frac{1}{2 \gamma \omega_{n}}\left(|\omega|-(1-\gamma) \omega_{n}\right)\right)\right] ; \text { if }(1-\gamma) \omega_{n} \leq|\omega| \leq(1+\gamma) \omega_{n} \\
0 ; \text { otherwise }
\end{array}\right.
$$

We can now define the Empirical Wavelet Transform $(\mathrm{EWT}), W_{f}(n, t)$ in the similar way as for the classic wavelet transform. By performing the inner product operation between the applied signal $f$, and with empirical wavelets we can obtain the detail coefficients as given in (9) and (10) respectively [10-11].

$$
W_{f}(n, t)=<f, \psi_{n}>=\int f(\tau) \psi_{n}(\tau-t) d \tau=\operatorname{IFFT}\left(F(\omega) \times \psi_{n}(\omega)\right)
$$

By performing the inner product operation between the applied signal $f$, and with empirical scaling functions we can obtain the approximation coefficients as given

$$
W_{f}(0, t)=<f, \phi_{1}>=\int f(\tau) \phi_{1}(\tau-t) d \tau=\operatorname{IFFT}\left(F(\omega) \times \phi_{1}(\omega)\right)
$$

Where $\phi_{1}(\omega)$ and $\psi_{n}(\omega)$ are defined by (7) and (8), respectively. The reconstruction is obtained by

$$
\begin{aligned}
& f(t)=W_{f}(0, t) * \phi_{1}(t)+\sum_{n=1}^{N} W_{f}(n, t) * \psi_{n}(t) \\
& f(t)=\operatorname{IFFT}\left(W_{f}(0, \omega) \times \phi_{1}(\omega)+\sum_{n=1}^{N} W_{f}(n, \omega) \times \psi_{n}(\omega)\right)
\end{aligned}
$$

Resulting this formalism, the empirical mode $f_{k}$, as defined 


$$
\begin{aligned}
& f_{0}(t)=W_{f}(0, t) * \phi_{1}(t) \\
& f_{k}(t)=W_{f}(k, t) * \psi_{k}(t)
\end{aligned}
$$

Where $*$ represents the convolution

This method works in the following Four steps.

1) Use FFT Algorithm to obtain the spectrum of the processed signal

2) Compute the local maxima of the spectrum

3) Detect the boundaries then segment the spectrum

4) Construct the empirical wavelets and decompose the signal into different components

\section{Hilbert Transform}

For a distorted input signal $x(n)$ contain $N$ frequency components, the $E W T(i, n)$ correspond to the $n^{\text {th }}$ sample of the extracted $i^{\text {th }}$ frequency component. As the extracted modes contain only one frequency component, The Hilbert transform (HT) can be used to estimate the instantaneous amplitude and frequency information.

The HT [14] of a real-valued function $E W T(i, t)=a_{i} \sin \left(2 \pi f_{i} t-\phi_{i}\right)$ computes its complex conjugate $E W T_{H}(i, t)$ determined by

$$
E W T_{H}(i, t)=\frac{1}{\pi} p \cdot v \cdot \int_{-\infty}^{\infty} \frac{E W T(i, \tau)}{t-\tau} d \tau
$$

Where (p.v.) is the Cauchy principle value of the singular integral. This orthogonal imaginary part of the original real signal is necessary to define its instantaneous phase. Consequently, the obtained analytic signal corresponding to $E W T(i, t)$ is defined as

$$
E W T_{a}(i, t)=E W T(i, t)+j E W T_{H}(i, t)=I A(i, t) e^{j \theta_{i}(t)}
$$

The instantaneous amplitude and instantaneous phase are

$$
\begin{aligned}
& I A(i, t)=\sqrt{(E W T(i, t))^{2}+\left(E W T_{H}(i, t)\right)^{2}} \\
& \theta_{i}(t)=\tan ^{-1}\left(\frac{E W T_{H}(i, t)}{E W T(i, t)}\right)
\end{aligned}
$$

Instantaneous frequency can be obtained from the phase function as by differentiating the Instantaneous phase

$$
I F(i, t)=\frac{1}{2 \pi} \frac{d \theta_{i}(t)}{d t}
$$

Accordingly the HT simply estimates the instantaneous parameters $I F(i, n)$ and $I A(i, n)$ of the signal $E W T(i, n)$.

\section{Time-Varying Power Quality Indices}

To describe the quality of the voltage or current certain parameters and their combinations are needed. These are termed as PQ indices and can be used to quantify the voltage or current quality. Time-varying PQ indices are the time-frequency distribution based indices, which illustrate the quality of nonstationary current (or) voltage signal. The indices are computed for each sample $(n)$ giving information of the signal with respect to 
the time, and so termed as instantaneous PQ indices [15-16]. The instantaneous frequency variation, instantaneous distortion energy index and instantaneous $\mathrm{K}$-factor help in the assessment of transient disturbances [16]. The PQ indices described in this paper are for the single phase voltage and current signals.

\subsection{Instantaneous Root Mean Square (iRMS)}

It is one of the most primitive and significant indices helpful for power computations, which is obtained from the instantaneous amplitude of all frequency components present in the signal [15].

$$
X_{r m s}(n)=\sqrt{\frac{1}{2} \sum_{i=1}^{N} I A(i, n)^{2}}
$$

Where $I A(i, n)$ represents instantaneous amplitude of $i^{\text {th }}$ frequency component and $\mathrm{N}$ is the total number of frequencies present in the input signal

\subsection{Instantaneous Fundamental Amplitude (iFA)}

This index is crucial in the recognition of magnitude associated disturbances like voltage sag, voltage swell, and interruption. It is obtained from the HT of the extracted time-varying fundamental frequency component of the signal.

$$
i F A(n)=I A(i, n) ; \forall f_{i} \approx 50 \mathrm{~Hz}
$$

\subsection{Instantaneous Frequency Variation (iFV)}

It helps in exploring the localization of frequency associated disturbances like harmonics, transients, and voltage fluctuation. The frequency of every component is weighted by its instantaneous energy reflecting dominant frequency component of the signal or elevated value for a disturbance with high frequencies [16].

$$
i F V(n)=\frac{\sum_{i=1}^{N} I F(i, n) \operatorname{IA}(i, n)^{2}}{\sum_{i=1}^{N} \operatorname{IA}(i, n)^{2}}
$$

Where $I F(i, n)$ is the instantaneous frequency of the $i^{\text {th }}$ frequency component obtained from the HT.

\subsection{Instantaneous Total Harmonic Distortion (iTHD)}

The most familiar index to evaluate the harmonic power relative to the fundamental frequency component is the total harmonic distortion (THD), its instantaneous calculation is achieved as

$$
i T H D(n)=\frac{\sqrt{\sum_{i=2}^{N} I A(i, n)^{2}}}{I A(1, n)}
$$

Where $I A(1, n)$ is understood to be fundamental frequency component.

\subsection{Instantaneous Normalized Distortion Energy Index (iNDEI)}

This PQ index is a portion of the disturbance energy to the total energy of the signal. Here, it is understood that $I A(1, n)$ represents the fundamental frequency component of the signal [16] 


$$
i N D E I(n)=\frac{\sqrt{\sum_{i=2}^{N} I A(i, n)^{2}}}{\sqrt{\sum_{i=1}^{N} I A(i, n)^{2}}}
$$

\subsection{Instantaneous K-Factor (iKF)}

It measures disturbance content of the signal in terms of squared normalized frequencies weighted by its energy [16]. It is analogous to iFV however it has more sensitiveness to frequencies as it is proportional to its square. For a normal signal, the value remains one.

$$
i K F(n)=\frac{\sum_{i=1}^{N} I F_{N}(i, n)^{2} I A(i, n)^{2}}{\sum_{i=1}^{N} I A(i, n)^{2}}
$$

Where $I F_{N}(i, n)=\frac{I F(i, n)}{50}$

\subsection{Instantaneous Form Factor (iFF)}

$\mathrm{iFF}$ is defined as the ratio of iRMS of the signal to the instantaneous mean of the signal [16]

$$
i F F(n)=\frac{X_{r m s}(n)}{\frac{2}{\pi} \sum_{i=1}^{N} I A(i, n)}
$$

\section{Results and Discussion}

In order to evaluate the performance of the EWT for estimation of time-varying PQ indices, Various disturbances as per IEEE standards encountered in power system such as Voltage Sag, Voltage Swell, Interruption, Flicker, Oscillatory transient ,Harmonics, Sag with Harmonic, Swell with Harmonic, Spike, Notch, have been considered for the analysis. First EWT decompose the distorted signal into Fundamental component and Disturbance components. As the extracted components hold only one frequency component, Hilbert transform is used to estimate the instantaneous amplitude and frequency information, from this information we can estimate Time-Varying PQ indices. In this work, Instantaneous root mean square, Instantaneous fundamental amplitude, Instantaneous frequency variation, Instantaneous total harmonic distortion, Instantaneous normalized distortion energy index, Instantaneous K-factor, Instantaneous form factor are suggested for power quality assessment.EWT- based Time-Varying power quality indices allow one to quantify the effects of disturbances with high-resolution and accuracy.

\subsection{Sag PQD Event}

The equation for Sag power Quality disturbance is given in Equation (27), and the parameters used in this equation are given in (28). Equation Model is [7]

$$
h(t)=\left[1-\alpha\left(u\left(t-t_{1}\right)-u\left(t-t_{2}\right)\right)\right] \sin \left(\omega_{b} t\right)
$$

Parameters $\quad 0.1 \leq \alpha \leq 0.9 ; T \leq t_{2}-t_{1} \leq 9 T ; \omega_{b}=2 \pi .50 ; T=1 / 50$ 
Sag type power Quality disturbance signal is shown in Figure 1.a.for the parameters $\alpha=0.9 ; t_{1}=0.05 ; t_{2}=0.15 ; f_{s}=10000$; the iRMS and iFA shown in Figure 1.b and 1.c. clearly replicate the voltage Sag. The iFA value can be used to identify the start time, end time, and thereby the duration of Sag accurately. The EWT-based indices iFV, iNDEI, iTHD and iKF, shown in Figure 1 remain constant throughout the signal except for the sag event duration.

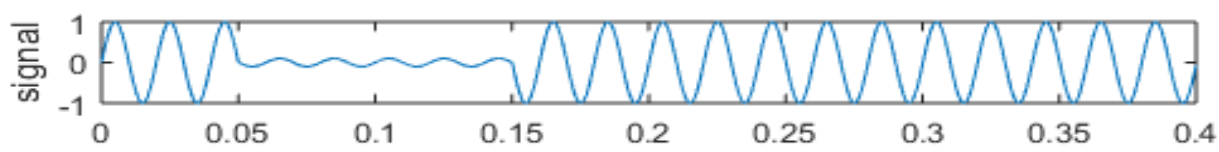

(a)

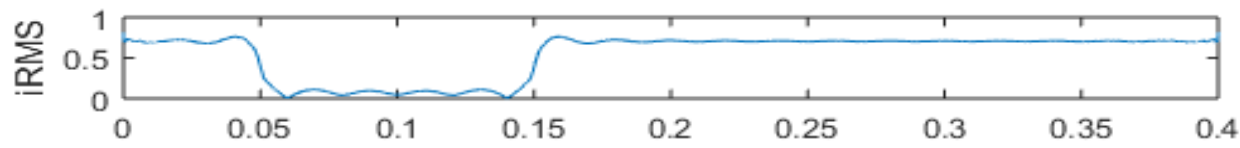

(b)

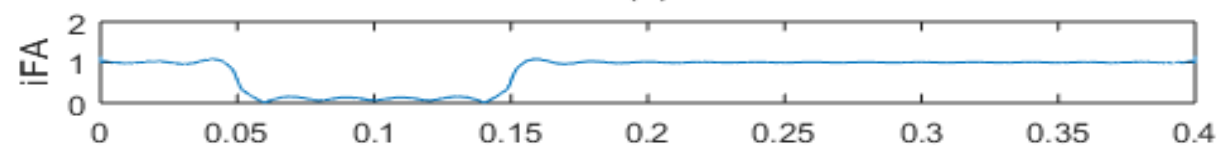

(c)

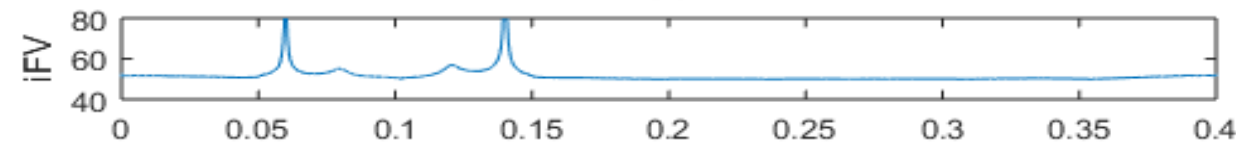

(d)

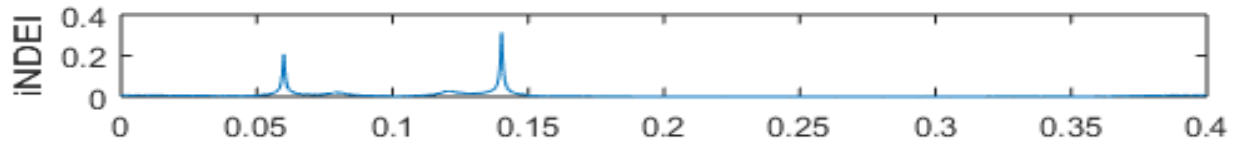

(e)

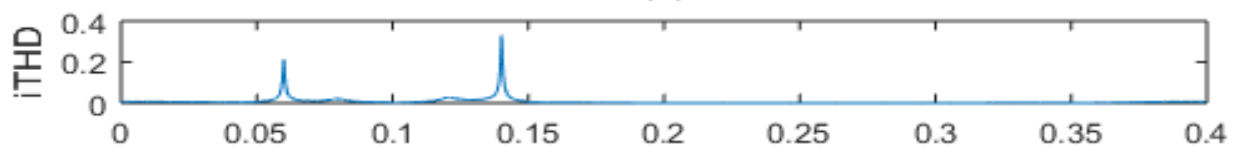

(f)

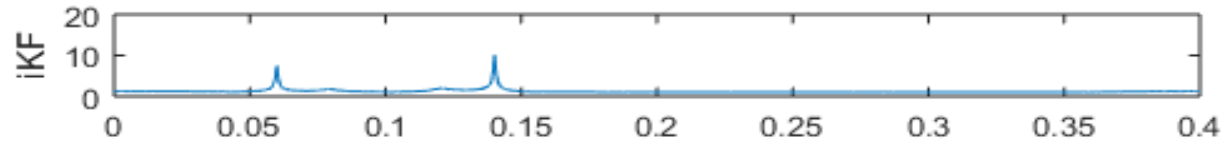

(g)

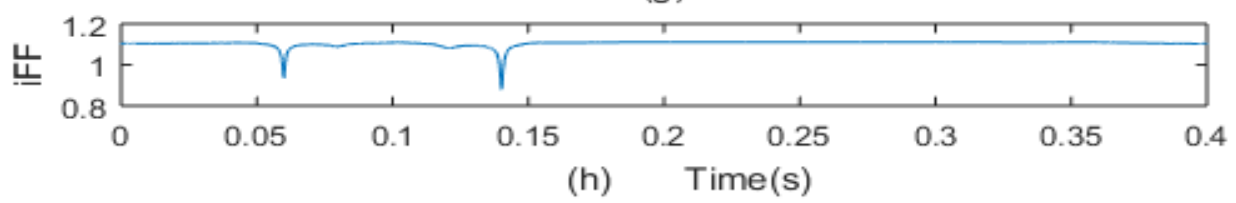

Figure 1. Analysis of Voltage Sag Signal and Its Indices

\subsection{Swell PQD Event}

The equation for Swell type power Quality disturbance is given in Equation (29), and the parameters used in this equation are given in (30).Equation Model is [7]

$$
h(t)=\left[1+\alpha\left(u\left(t-t_{1}\right)-u\left(t-t_{2}\right)\right)\right] \sin \left(\omega_{b} t\right)
$$


Parameters $\quad 0.1 \leq \alpha \leq 0.8 ; T \leq t_{2}-t_{1} \leq 9 T ; \omega_{b}=2 \pi .50 ; T=1 / 50$

Swell PQD signal is shown in Figure 2.a for the parameters $\alpha=0.8 ; t_{1}=0.05 ; t_{2}=0.15 ; f_{s}=10000$;

The iRMS and iFA shown in Figure 2.b and 2.c. clearly replicate the voltage swell. The iFA value can be used to identify the start time, end time, and thereby the duration of swell accurately. The EWT-based indices iFV, iNDEI, iTHD and iKF, shown in Figure 2 remain constant throughout the signal except for the swell event duration.

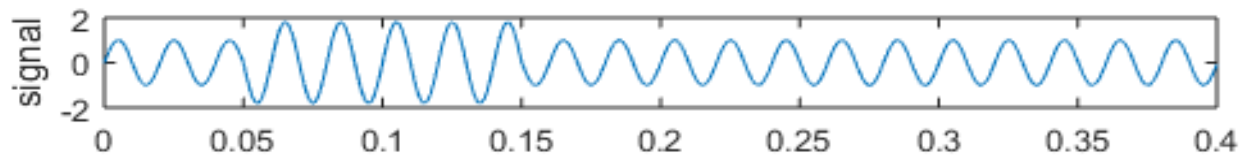

(a)

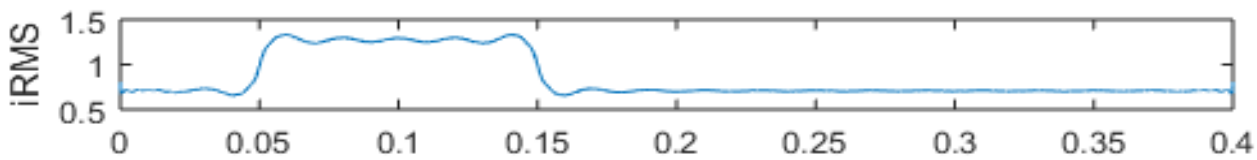

(b)

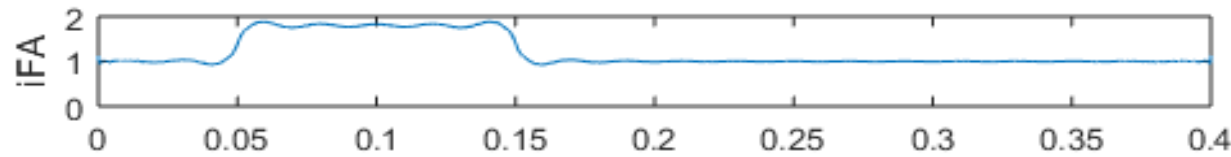

(c)

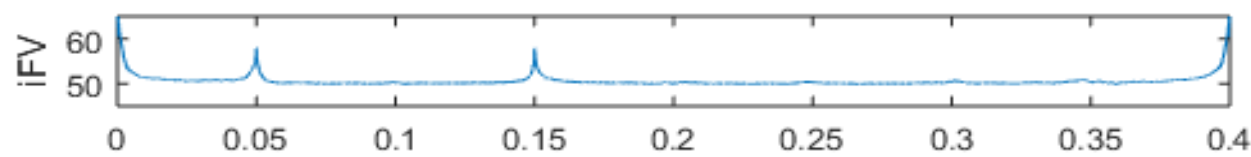

(d)

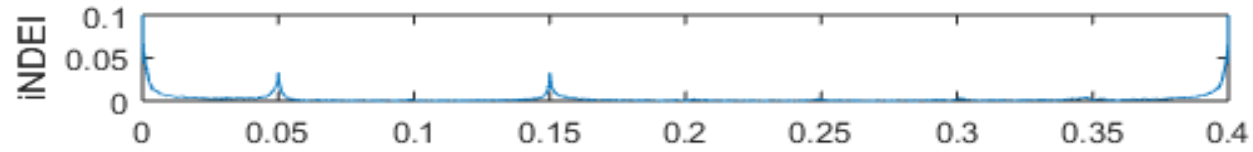

(e)

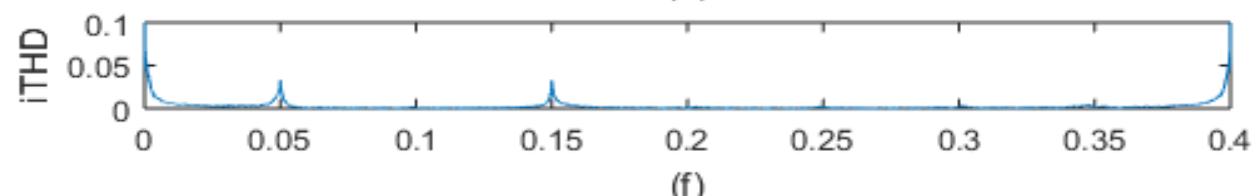

(f)

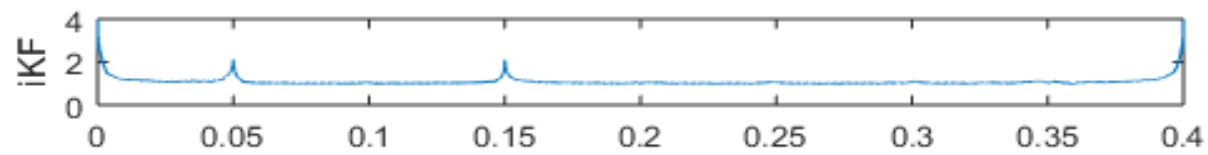

(g)

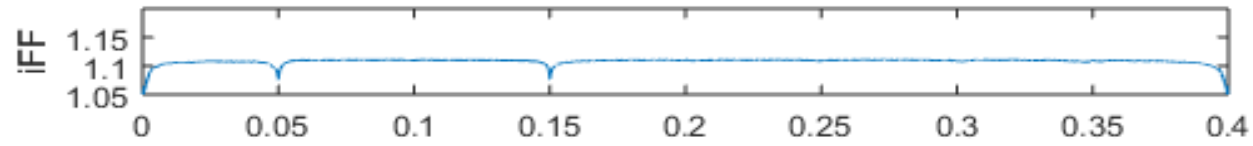

(h) Time(s)

Figure 2. Analysis of Voltage Swell Signal and Its Indices

\subsection{Interruption}

The equation for Interruption type power Quality disturbance is given in Equation (31), and the parameters used in this equation are given in (32).Equation Model is [7]. 


$$
h(t)=\left[1-\alpha\left(u\left(t-t_{1}\right)-u\left(t-t_{2}\right)\right)\right] \sin \left(\omega_{b} t\right)
$$

Parameters $\quad 0.9 \leq \alpha \leq 1 ; T \leq t_{2}-t_{1} \leq 9 T ; \omega_{b}=2 \pi .50 ; T=1 / 50$

Interruption Power Quality Disturbance signal is shown in Figure 3. a.for the parameters $\alpha=1 ; t_{1}=0.05 ; t_{2}=0.15 ; f_{s}=10000$; the iRMS and iFA shown in Figure 3.b and 3.c. clearly replicate the Interruption. The iFA value can be used to identify the start time, end time, and thereby the duration of Interruption accurately. The EWT-based indices iFV, iNDEI, iTHD, iKF, and iFF shown in Figure 3 remain constant throughout the signal except for the Interruption event duration.

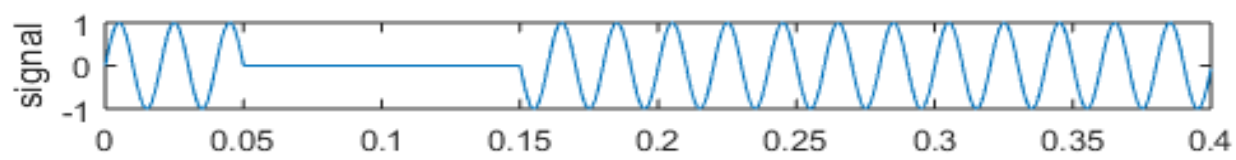

(a)

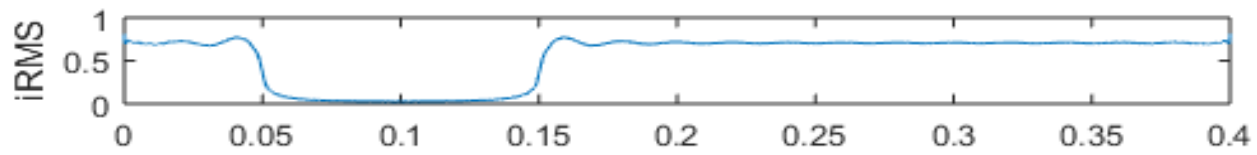

(b)

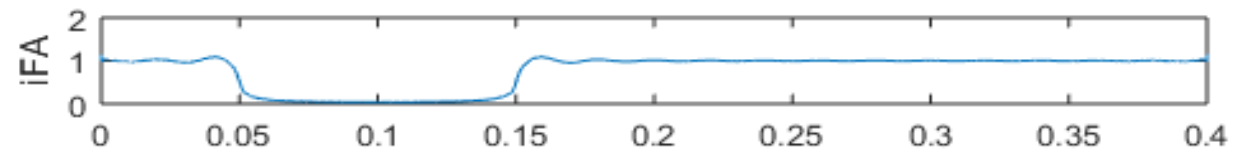

(c)

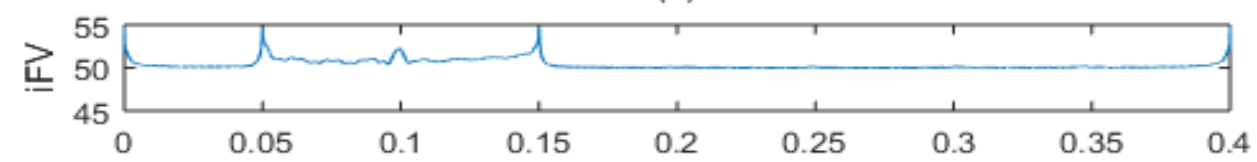

(d)

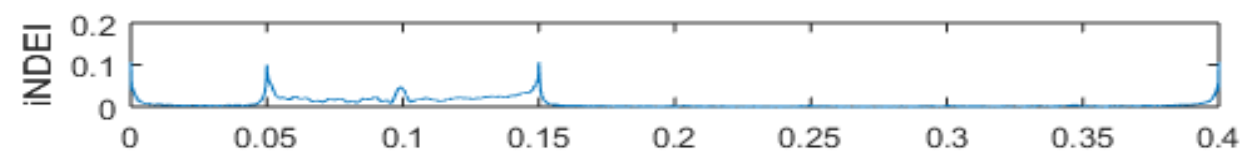

(e)

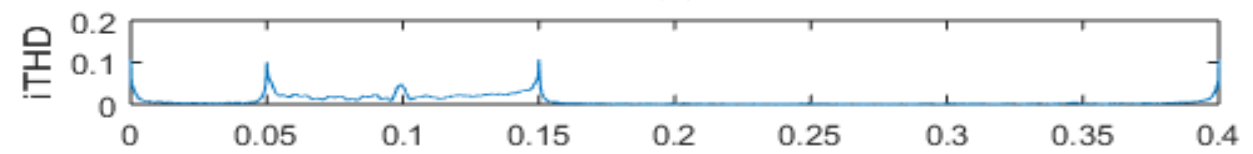

(f)

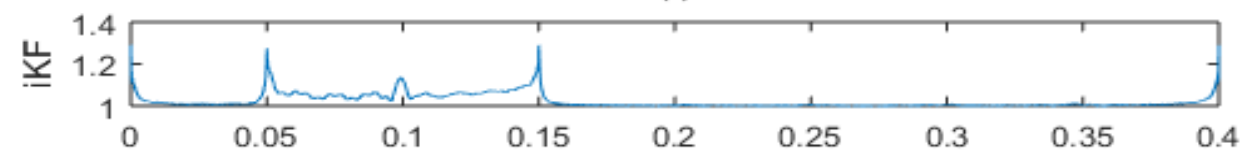

(g)

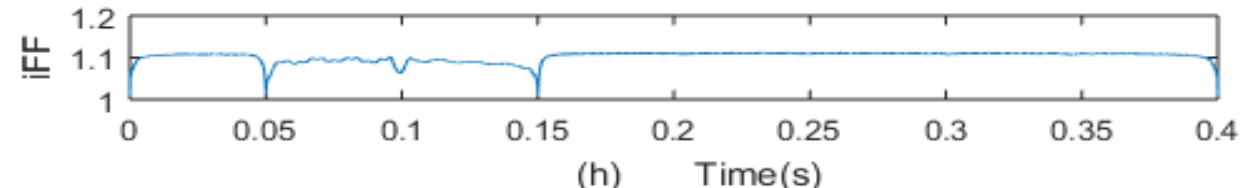

Figure 3. Analysis of Interruption Signal and Its Indices 


\subsection{Flicker}

Flicker is caused by the continuous and rapid variation of the system load. The presence of voltage flicker is characterized by amplitude modulation of the fundamental sine wave with a subharmonic frequency of less than $20 \mathrm{~Hz}$ frequency and less than $20 \%$ in magnitude. The equation for Flicker type power Quality disturbance is given in Equation (33), and the parameters used in this equation are given in (34). Equation Model is [7]

$$
h(t)=[1+\alpha \sin (2 \pi \beta t)] \sin \left(\omega_{b} t\right)
$$

Parameters $0.1 \leq \alpha \leq 0.2 ; 5 H Z \leq \beta \leq 20 H Z ; \omega_{b}=2 \pi .50$;

Flicker PQD signal is shown in Figure 4.a., for the parameters $\alpha=0.2 ; \beta=20 ; f_{s}=10000$;

For Flicker type signal the EWT-based indices iRMS, iFA, iFV, iNDEI, iTHD, iKF, and iFF shown in Figure 4, remains constant throughout the signal.

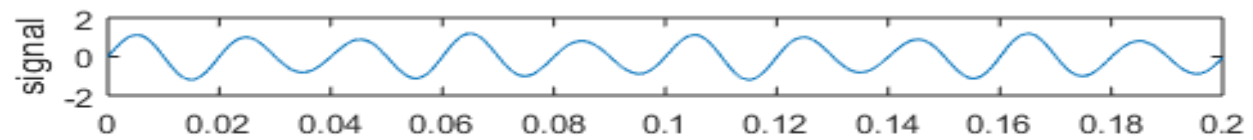

(a)

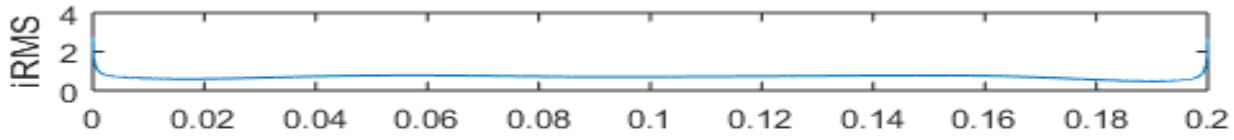

(b)

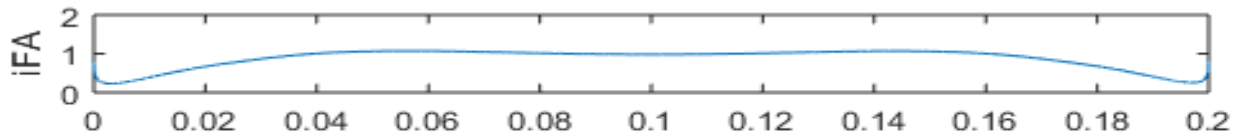

(c)

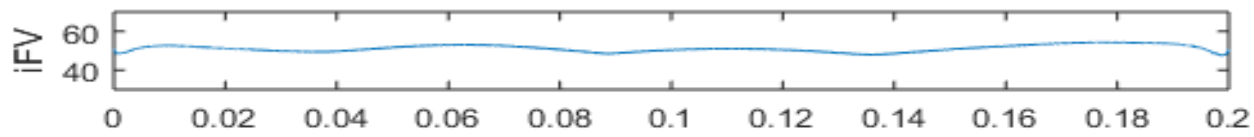

(d) Time (s)

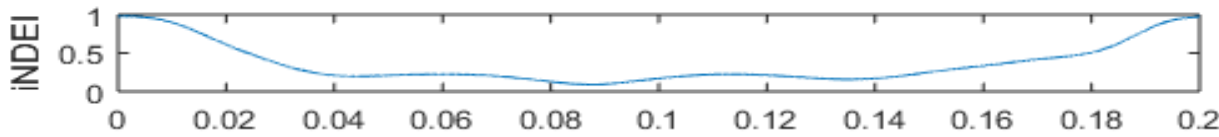

(e)

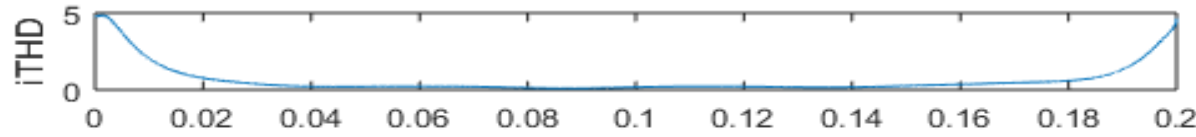

(f)

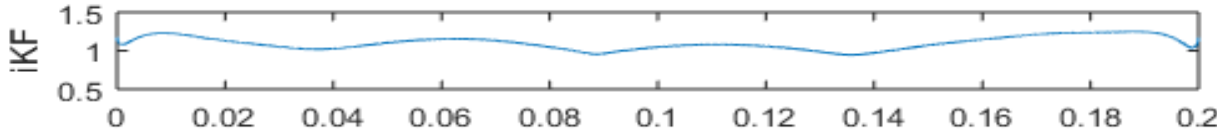

(g)

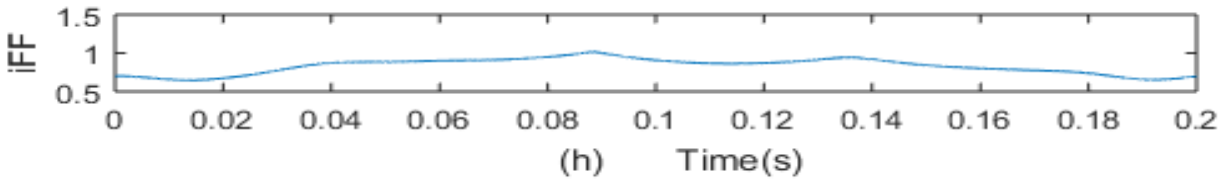

Figure 4. Analysis of Flicker Signal and Its Indices 


\subsection{Oscillatory Transient PQD Event}

The equation for Oscillatory transient type power Quality disturbance is given in (35), and the parameters used in this equation are given in (36).Equation Model is [7]

$$
h(t)=\sin \left(\omega_{b} t\right)+\alpha \exp \left(-\left(t-t_{1}\right) / \tau\right)\left(u\left(t-t_{1}\right)-u\left(t-t_{2}\right)\right) \sin \left(2 \pi f_{n} t\right)
$$

Parameters $0.1 \leq \alpha \leq 0.8 ; 0.5 T \leq t_{2}-t_{1} \leq 3 T ; 300 H Z \leq f_{n} \leq 900 H Z ; 8 m s \leq \tau \leq 40 m s ; \omega_{b}=2 \pi .50 ; T=1 / 50$

Oscillatory transient PQD signal is shown in Figure 5.a.for the parameters $\alpha=0.8 ; f_{n}=300 ; t_{1}=0.041 ; t_{2}=0.071 ; f_{s}=10000$; EWT has been applied to analyze and estimate the indices for quantizing the transient. The signal contains high frequencies during the transient, consequently, the EWT-based iFV, iTHD, and iKF index values are very high during the transient. The significant rise in values of iRMS, iFV, iTHD and iKF for a short duration clearly indicate the presence of transient in the signal.EWT-based time varying indices can extract time-varying features with exact instants of the transient. These indices permit us to record the initial time duration of the transient occurred.

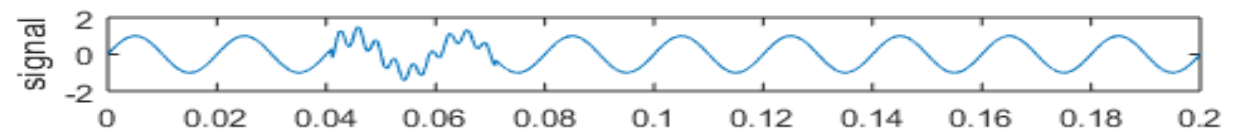

(a)

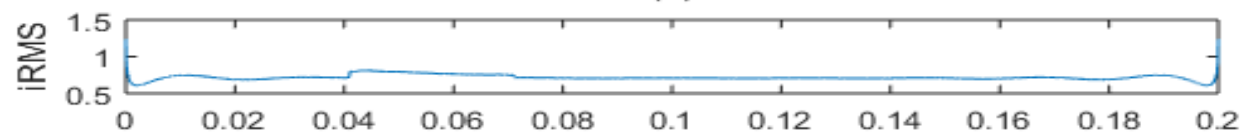

(b)

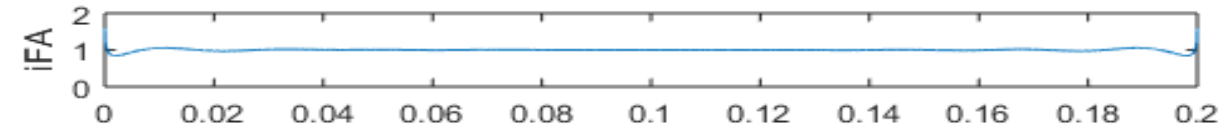

(c)
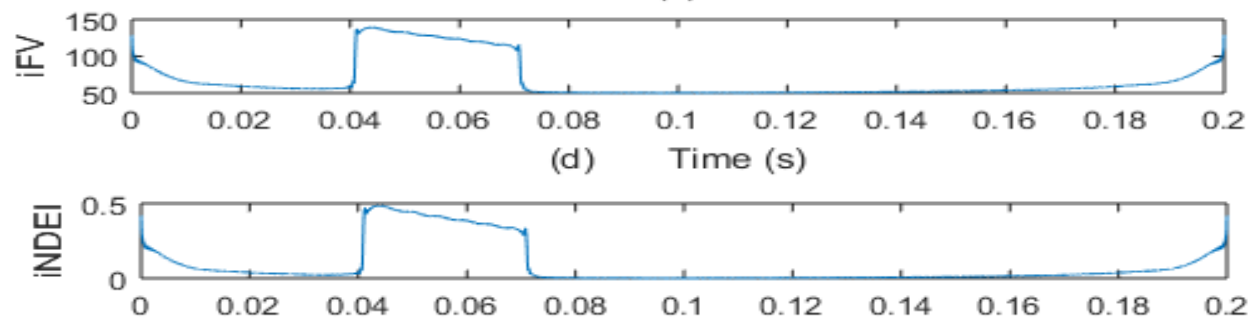

(e)

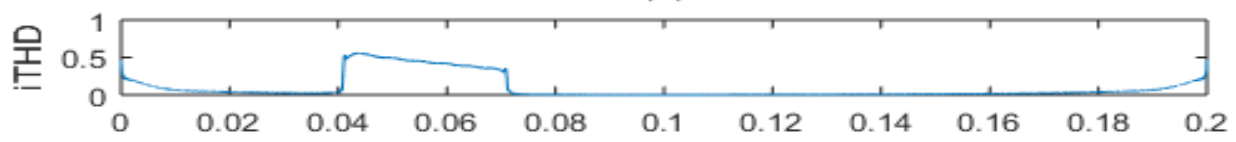

(f)

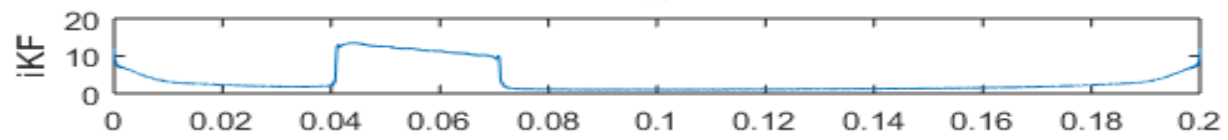

(g)

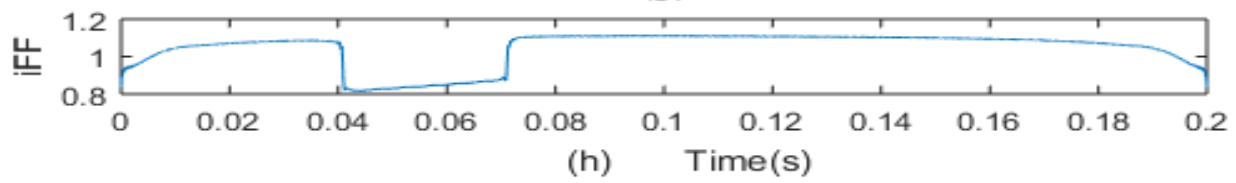

Figure 5. Analysis of Oscillatory Transient Signal and Its Indices 


\subsection{Harmonic PQD Event}

The equation for Harmonic type power Quality disturbance is given in Equation (37), and the parameters used in this equation are given in (38).Equation Model is [7]

$$
h(t)=\alpha_{1} \sin \left(\omega_{b} t\right)+\alpha_{3} \sin \left(3 \omega_{b} t\right)+\alpha_{5} \sin \left(5 \omega_{b} t\right)+\alpha_{7} \sin \left(7 \omega_{b} t\right)
$$

Parameters $0.05 \leq \alpha_{3}, \alpha_{5}, \alpha_{7} \leq 0.15 ; \Sigma\left(\alpha_{i}\right)^{2}=1 ; \omega_{b}=2 \pi .50$;

Harmonic type PQD signal is shown in Figure 6.a.for the parameters $f_{S}=10000 ; \alpha=0.5 ; \alpha_{3}=\alpha_{5}=\alpha_{7}=0.15$; The analysis reveals that the Harmonic PQD signal does not contain any inter harmonics but has only harmonics up to $7^{\text {th }}$ order. Hence all the time varying indices are constant.

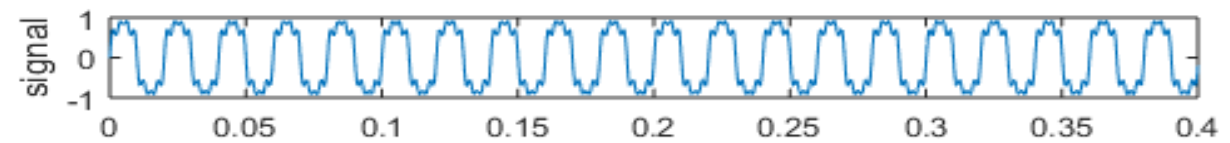

(a)

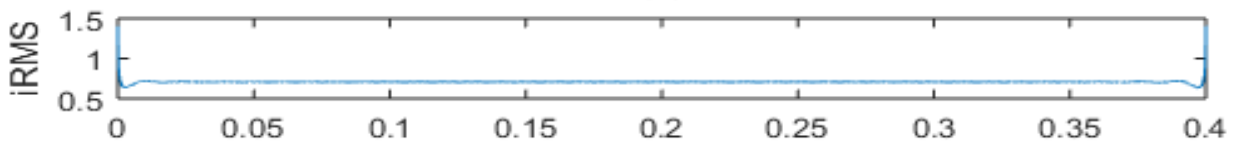

(b)

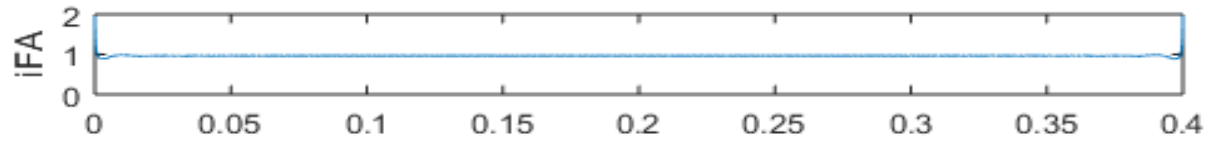

(c)

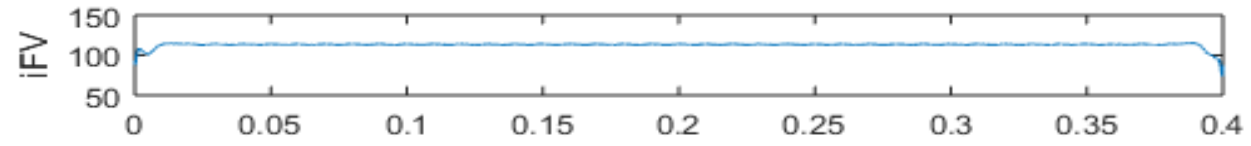

(d) Time (s)

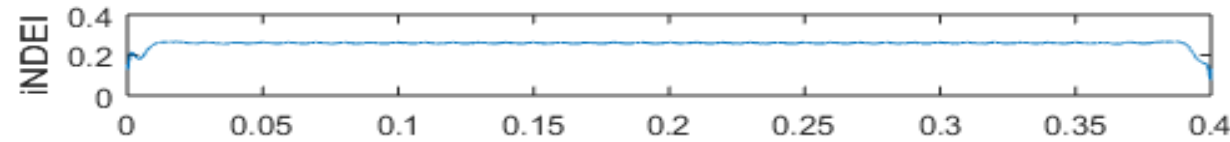

(e)

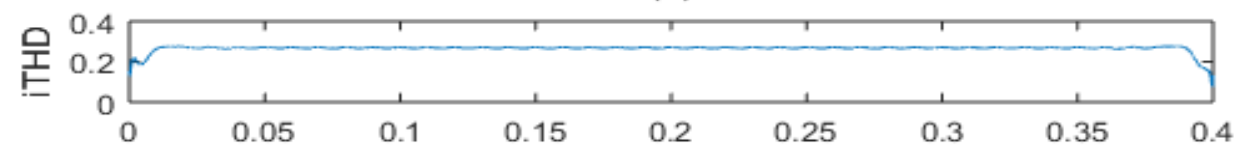

(f)

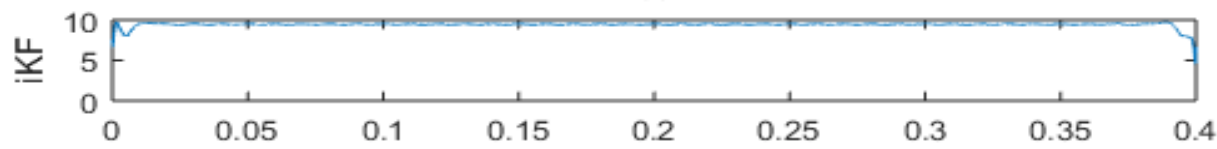

(g)

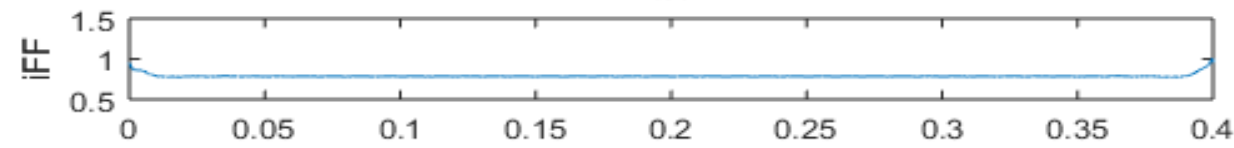

(h) Time(s)

Figure 6. Analysis of Harmonics Signal and Its Indices 


\subsection{Sag+Harmonics}

The equation for Sag with Harmonic type power Quality disturbance is given in Equation (39), and the parameters used in this equation are given in (40) [7]

$$
\begin{aligned}
& h(t)=\left[1-\alpha\left(u\left(t-t_{1}\right)-u\left(t-t_{2}\right)\right)\right]\left[\alpha_{1} \sin \left(\omega_{b} t\right)+\alpha_{3} \sin \left(3 \omega_{b} t\right)+\alpha_{5} \sin \left(5 \omega_{b} t\right)\right] \\
& 0.1<\alpha \leq 0.9 ; T \leq t_{2}-t_{1} \leq 9 T ; 0.05 \leq \alpha_{3}, \alpha_{5} \leq 0.15 ; \Sigma\left(\alpha_{i}\right)^{2}=1 ; \omega_{b}=2 \pi .50 ; T=1 / 50,
\end{aligned}
$$

Sag with Harmonic type PQD signal is shown in Figure 7.a.for the parameters $\alpha=0.9 ; \alpha_{3}=\alpha_{5}=\alpha_{7}=0.15 ; t_{1}=0.05 ; t_{2}=0.15 ; f_{s}=10000$; The iRMS and iFA shown in Figure 7.b, and 7.c., clearly replicate the voltage sag. The iFA value can be used to identify the start time, end time, and thereby the duration of Sag accurately. The EWTbased indices iFV, iNDEI, iTHD and iKF, shown in Figure 7 remain constant throughout the signal except for the Sag event duration.

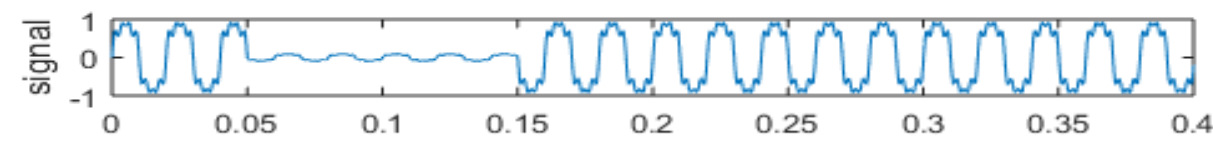

(a)

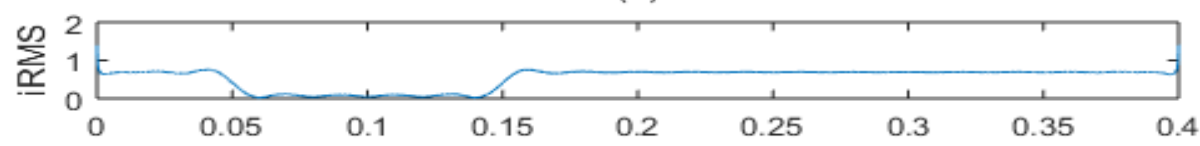

(b)

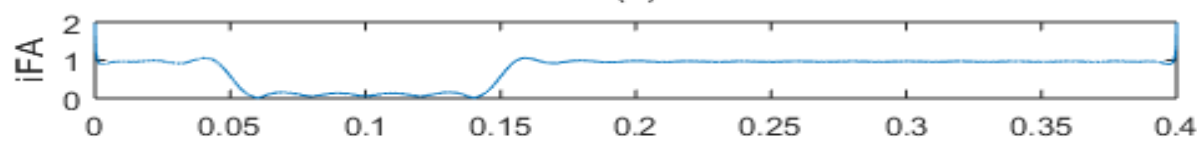

(c)

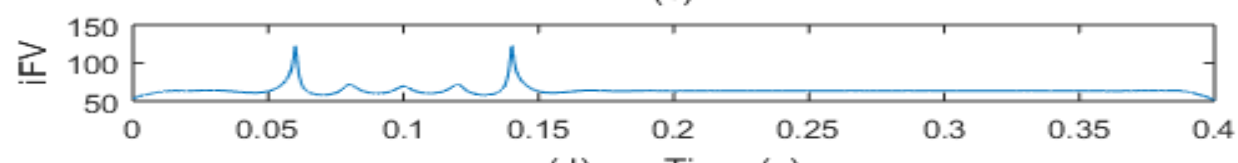

(d) Time (s)

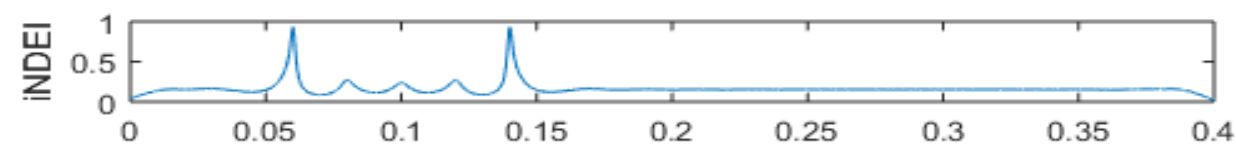

(e)

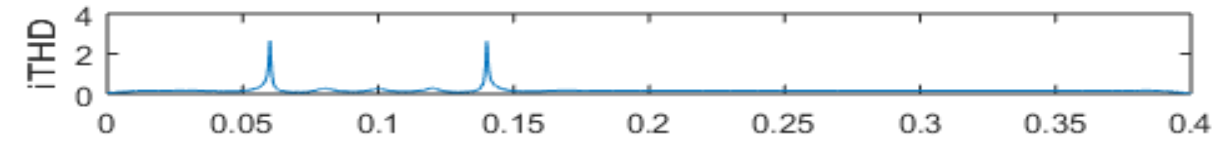

(f)

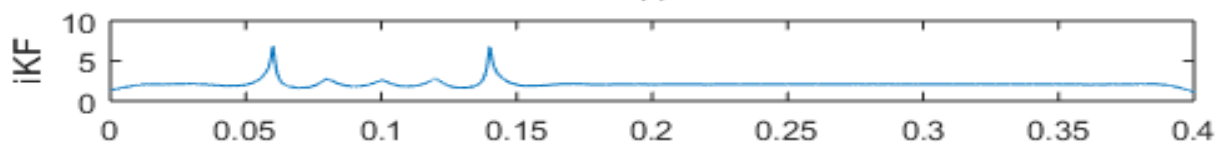

(g)

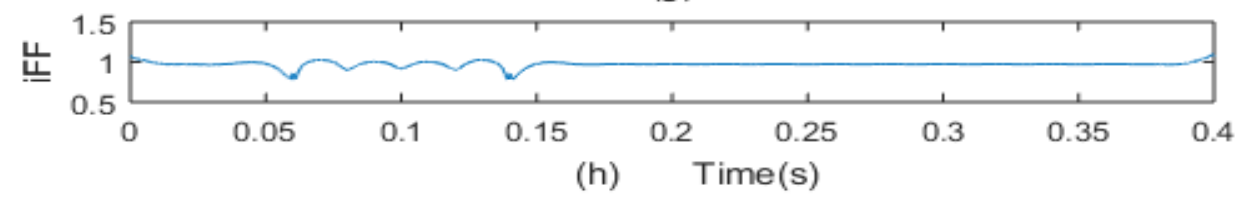

Figure 7. Analysis of Sag with Harmonics Signal and Its Indices 


\subsection{Swell+Harmonics Wave}

The equation for Swell with Harmonic type power Quality disturbance is given in Equation (41), and the parameters used in this equation are given in (42) [7]

$$
h(t)=\left[1+\alpha\left(u\left(t-t_{1}\right)-u\left(t-t_{2}\right)\right)\right]\left[\alpha_{1} \sin \left(\omega_{b} t\right)+\alpha_{3} \sin \left(3 \omega_{b} t\right)+\alpha_{5} \sin \left(5 \omega_{b} t\right)\right]
$$

Parameters $0.1<\alpha \leq 0.8 ; T \leq t_{2}-t_{1} \leq 9 T ; 0.05 \leq \alpha_{3}, \alpha_{5} \leq 0.15 ; \Sigma\left(\alpha_{i}\right)^{2}=1 ; \omega_{b}=2 \pi .50$;

Swell with Harmonic type PQD signal is shown in Figure 8.a.for the parameters $\alpha=0.5 ; \alpha_{3}=\alpha_{5}=\alpha_{7}=0.15 ; t_{1}=0.05 ; t_{2}=0.15 ; f_{s}=10000 ;$ The iRMS and iFA shown in Figure 8.b, and 8.c. clearly replicate the voltage swell. The iFA value can be used to identify the start time, end time, and thereby the duration of swell accurately. The EWT-based indices iFV, iNDEI, iTHD and iKF, shown in Figure 8 remain constant throughout the signal except for the Swell event duration.

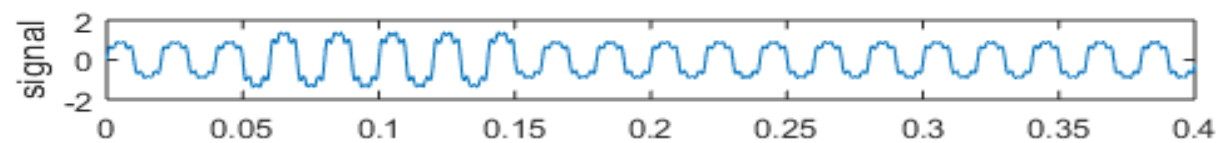

(a)

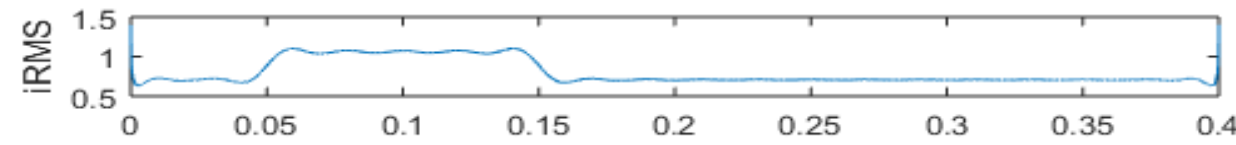

(b)

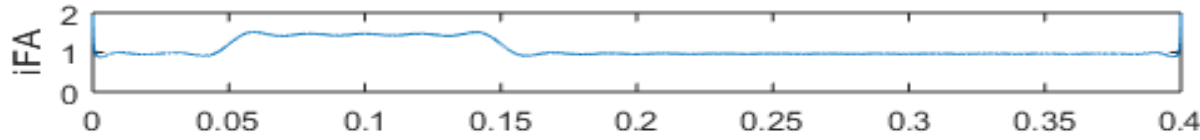

(c)

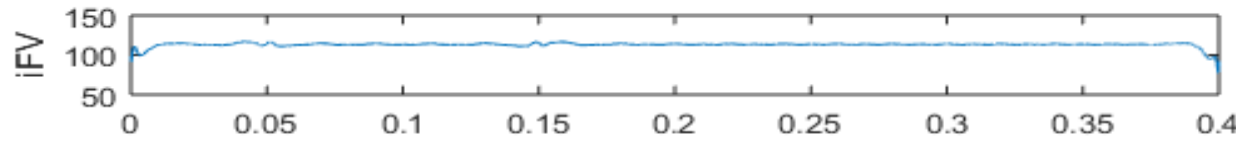

(d) Time (s)

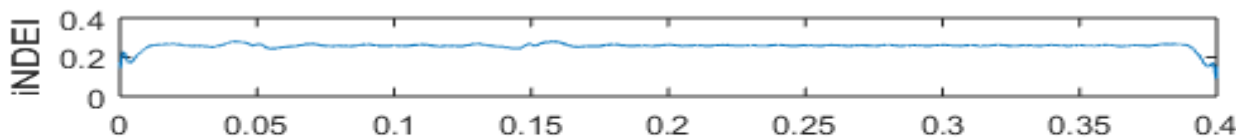

(e)

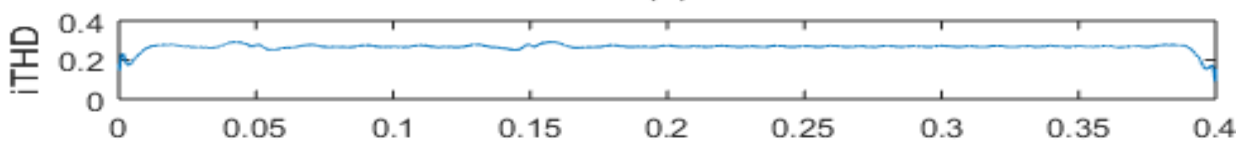

(f)

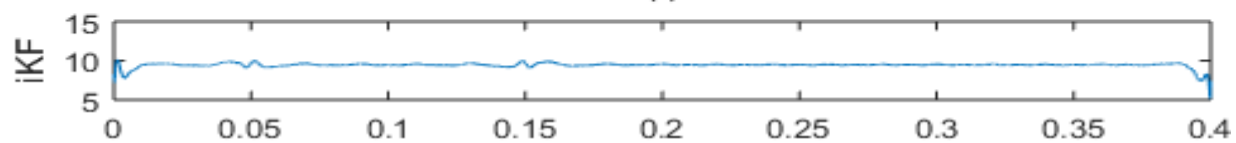

(g)

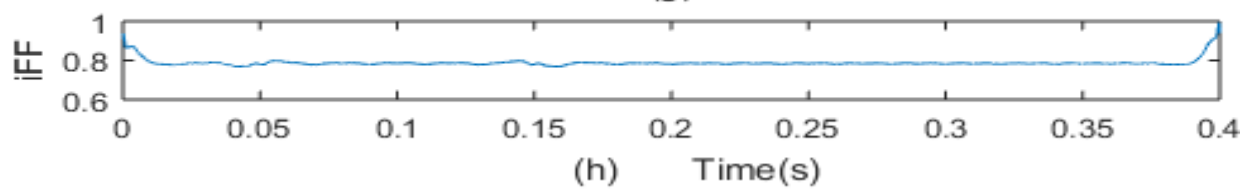

Figure 8. Analysis of Swell with Harmonics Signal and Its Indices 


\subsection{Notch PQD Event}

The equation for Notch type power Quality disturbance is given in Equation (43), and the parameters used in this equation are given in (44) [7]

$$
h(t)=\sin \left(\omega_{b} t\right)-\operatorname{sign}\left(\sin \left(\omega_{b} t\right)\right)\left\{\sum_{n=0}^{9} k\left[u\left(t-\left(t_{1}+0.02 n\right)\right)-u\left(t-\left(t_{2}+0.02 n\right)\right)\right]\right\}
$$

Parameter $0.1 \leq k \leq 0.4 ; 0 \leq t_{1}, t_{2} \leq 0.5 T ; 0.01 T \leq t_{2}-t_{1} \leq 0.05 T$

Notch Power Quality Disturbance signal is shown in Figure 9.a.for the parameters $k=0.4 ; t_{1}=0.0029 ; t_{2}=t_{1}+0.0005 ; f_{s}=10000$; The iFA is constant throughout the signal and the significant rise in values of iRMS, iFV, iNDEI and iKF for a short duration clearly indicate the presence of Notches in the signal.EWT-based time varying indices can extract time-varying features with exact instants of the notches.

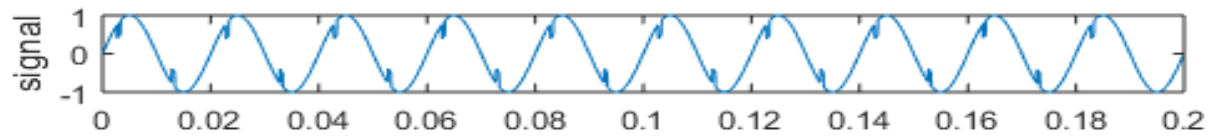

(a)

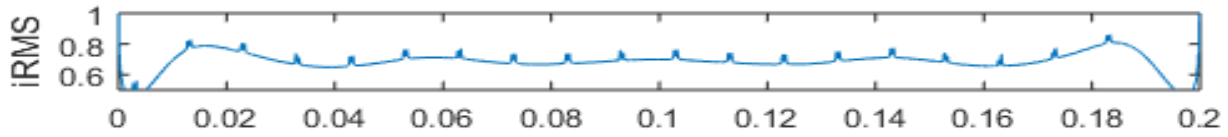

(b)

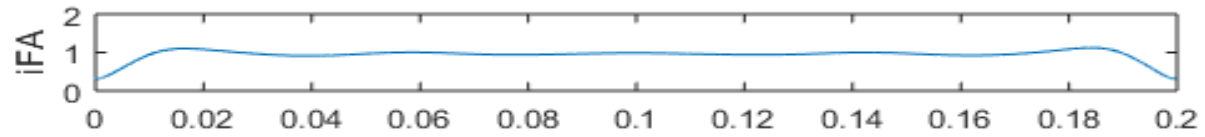

(c)
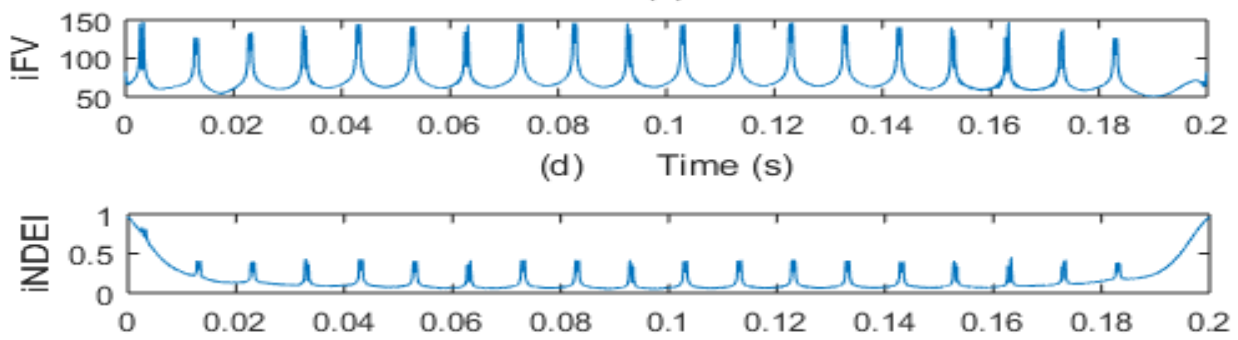

(e)

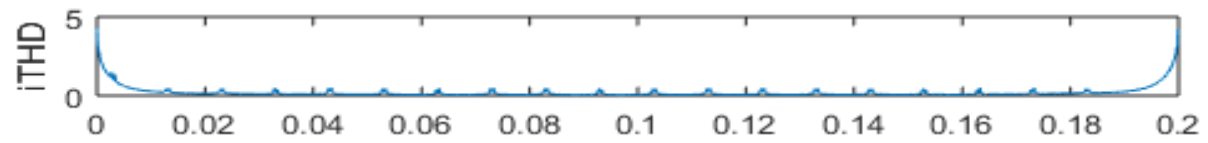

(f)

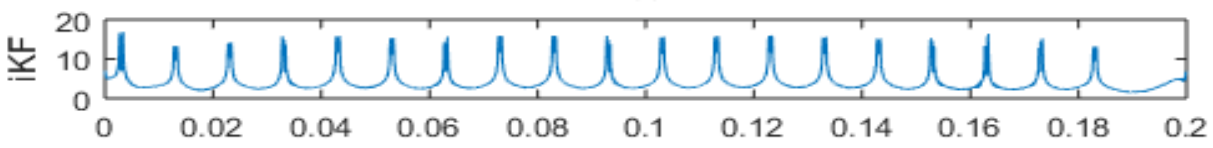

(g)

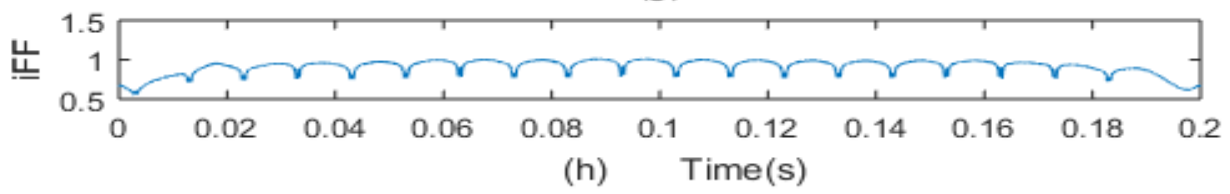

Figure 9. Analysis of Notch Signal and Its Indices 


\subsection{Spike PQD Event}

he equation for Spike type power Quality disturbance is given in Equation (45), and the parameters used in this equation are given in (46) [7]

$$
h(t)=\sin \left(\omega_{b} t\right)+\operatorname{sign}\left(\sin \left(\omega_{b} t\right)\right)\left\{\sum_{n=0}^{9} k\left[u\left(t-\left(t_{1}+0.02 n\right)\right)-u\left(t-\left(t_{2}+0.02 n\right)\right)\right]\right\}
$$

Parameters

$$
0.1 \leq k \leq 0.4 ; 0 \leq t_{1}, t_{2} \leq 0.5 T ; 0.01 T \leq t_{2}-t_{1} \leq 0.05 T ; \omega_{b}=2 \pi .50 ; T=1 / 50
$$

Spike Power Quality Disturbance signal is shown in Figure 10.a.for the parameters $k=0.4 ; t_{1}=0.0029 ; t_{2}=t_{1}+0.0005 ; f_{s}=10000$; The iFA is constant throughout the signal and the significant rise in values of iRMS, iFV, iNDEI and iKF for a short duration clearly indicate the presence of Spikes in the signal.EWT-based time varying indices can extract time-varying features with exact instants of the Spikes.

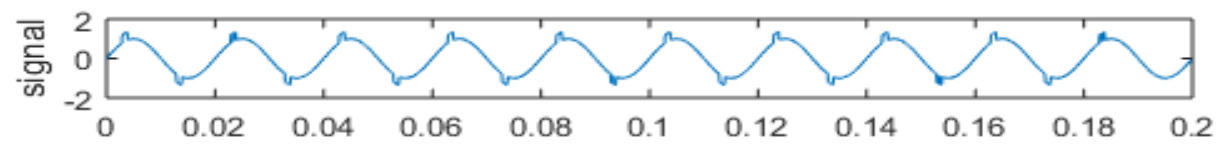

(a)

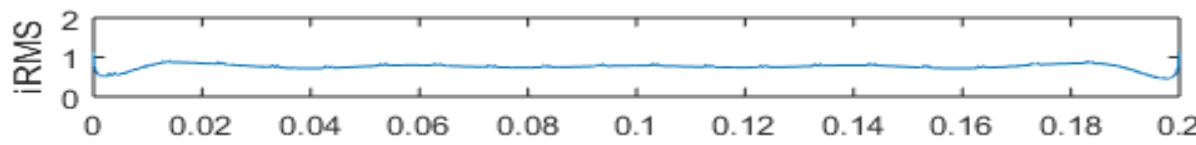

(b)

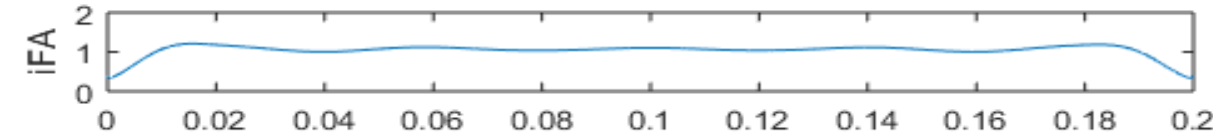

(c)

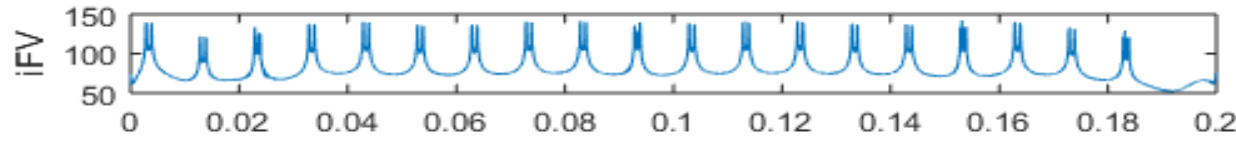

(d) Time (s)

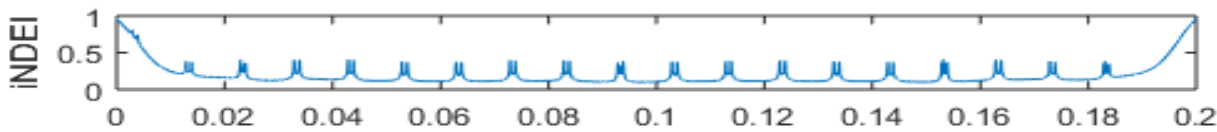

(e)

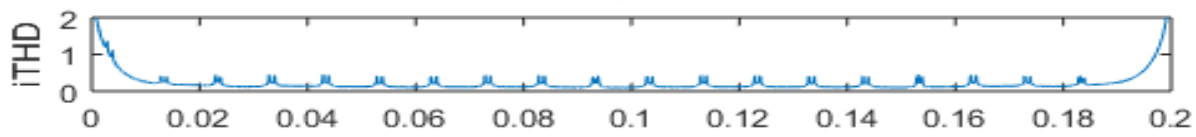

(f)

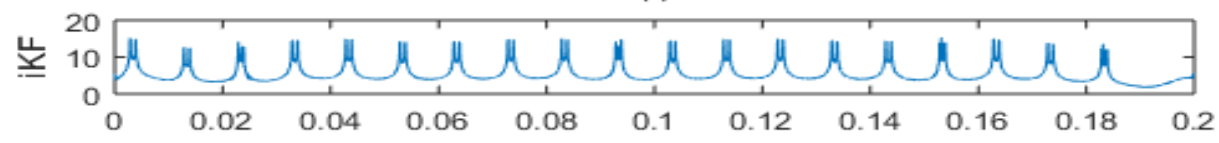

(g)

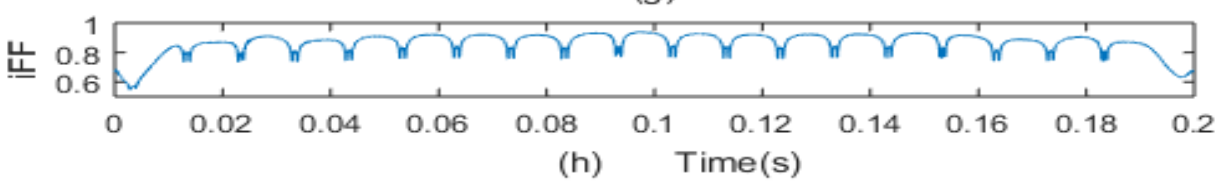

Figure 10. Analysis of Spike Signal and Its Indices 


\section{Conclusions}

This paper presents the estimation of time-varying PQ indices using a EWT for accurate assesment of PQ disturbances.Results of all the case studies revealed that the characteristics of the signal can be predicted by observing the EWT -based time -varying PQ indices. Instantaneous fundamental amplitude index can be used in the identification of magnitude related disturbances like voltage sag, voltage swell and interuption. Instantaneous fundamental variation can be used in exploring the localization of frequency related disturbances like transients, harmonics.The accuracy of these indices plays a major role in the detection of disturbances with extreme intensities. The analysis of PQDisturbance signals proves that the EWT can be sucessfully employed for real-time estimation for PQindices.

\section{References}

[1] IEEE Recommended Practice and Requirements for Harmonic Control in Electric Power Systems, IEEE Standard 519-1992, (1993) Apr.

[2] Definitions for the Measurement of Electric Quantities under Sinusoidal, on-Sinusoidal, Balanced, or Unbalanced conditions, IEEE Standarad 1459-2010, (2000) Jan.

[3] R. Flores, "Signal processing tools for power quality event classification", Lic. Eng. thesis, School Elect. Eng, Chalmers Univ. Technol, Göteborg, Sweden, (2003).

[4] M. Bollen and I. Yu-HuaaGu, "Signal Processing of Power Quality Disturbances", Hoboken, NJ, USA Wiley, (2006).

[5] D. Granados-Lieberman, R. J. Romero-Troncoso, R. A. Osornio-Rios, A. Garcia-Perez and E. CabalYepez, "Techniques and methodologies for power quality analysis and disturbances classification in power systems: a review," IET Generation, Transmission Distribution, vol. 5, no. 4, (2011), pp. 519529.

[6] S. Santoso, W. M. Grady, E. J. Powers, J. Lamoree and S. C. Bhatt, "Characterization of Distribution Power Quality Events with Fourier and Wavelet Transforms", IEE Transactions on power Delivery, vol. 15, no. 1, pp. 247-254.

[7] C. Y. Lee, and Y. X. Shen, "Optimal feature selection for power-quality disturbances Classification", IEEE Transactions on Power Delivery, vol. 26, no. 4, (2011), pp. 2342-2351.

[8] R. A. Brown, M. L. Lauzon and R. Frayne, "A general description of linear time-frequency transforms and formulation of a fast, invertible transform that samples the continuos S-transform spectrum nonredundantly", IEEETans.Signal Process, vol. 58, no. 1, (2010), pp. 281-290.

[9] A. Bracale, G. Capinelli, I. Y. -H. Gu and M. H. J.Bollen, "A new joint sliding-window ESPRIT and DFT scheme for waveform distortion assessment in power systems", Electr,Power Syst.Res. vol. 88, (2012), pp. 112-120.

[10] J. Gilles, "Empirical wavelet transform", IEEE Trans. Signal Process., vol. 61, no. 16, (2013) Aug., pp. 3999-4010.

[11] J. Gilles, G, Tran and S, Osher, "2D Empirical Transforms. Wavelets, Ridgelets and Curvelets Revisited", SIAM Journal on Imaging Sciences, vol. 7, no. 1, (2014), pp. 157-186.

[12] K. Thirumala, A. C. Umarikar and T. Jain, "Estimation of Single-Phase and Three-Phase Power-Quality Indices Using Empirical Wavelet Transform”, IEEE Transactions on Power Delivery, vol. 30, no. 1, (2015), pp. 445-454.

[13] L. Prakash, S. Mohan and K. P. Soman, "Accurate Frequency Estimation Method Based on Basis Approach and Empirical Wavelet Transform", Proceedings of the Second International Conference on Computer and Communication Technologies. Springer India, (2016).

[14] S. L. Hahn, Hilbert, "Transform in Signal Processing”, Artech House, London, (1996).

[15] Biswal, Milan, and P. K. Dash, "Estimation of time-varying power quality indices with an adaptive window-based fast generalized S-transform”, IET Science, Measurement \& Technology, vol. 6, no. 4, (2012), pp. 189-197.

[16] Y. -J. Shin, "Power quality indices for transient disturbances", IEEE Transactions on Power Delivery, vol. 21, no. 1, (2006), pp. 253-261. 


\section{Authors}

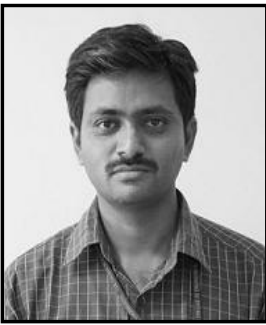

G. Ravi Shankar Reddy is presently working as an Associate Professor in the Department of ECE, CVR College of Engineering, Hyderabad, India. He received the B.Tech Degree from Jawaharlal Nehru Technological University, Hyderabad, India, in 2001.He received the M.Tech Degree from Osmania University, Hyderabad, India, in 2004.Presently He is pursuing his Ph.D. from JNTUA, Anantapur, India, under the guidance of Dr.Rameshwar Rao. His Research area is Non-Stationary Signal Processing.

Dr. Rameshwar Rao was Ex-Vice Chancellor of Jawaharlal Nehru Technological University, Hyderabad, India. He guided several PhD students in the area of VLSI and Signal processing. He has more than 50 publications in various National and International Journals .His research interests are in VLSI and Signal processing. 
International Journal of Signal Processing, Image Processing and Pattern Recognition Vol. 9, No. 11, (2016) 